\title{
Utilização de antimicrobianos em uma Unidade de Terapia Intensiva Neonatal: um estudo transversal retrospectivo
}

\author{
Use of antimicrobials in a Neonatal Intensive Care Unit: a retrospective cross-sectional study \\ Utilización de antimicrobianos en una Unidad de Terapia Intensiva Neonatal: un estudio
}

transversal retropectivo

Recebido: 08/01/2021 | Revisado: 10/01/2021 | Aceito: 12/01/2021 | Publicado: 15/01/2021

Cinara Rejane Viana Oliveira

ORCID: https://orcid.org/0000-0002-4835-2481

Universidade do Estado da Bahia, Brasil

E-mail: cinara-rejane@hotmail.com

Izamar Nunes Macedo

ORCID: http://orcid.org/0000-0001-8265-8225

Universidade Federal da Bahia, Brasil

E-mail:izamar.macedo@gmail.com

Maria Teresita Bendicho

ORCID: http://orcid.org/0000-0001-8234-1199

Universidade do Estado da Bahia, Brasil

E-mail:mtbendicho@gmail.com

Rosa Malena Fagundes Xavier

ORCID: http://orcid.org/0000-0002-3203-8949

Universidade do Estado da Bahia, Brasil

E-mail:rxavier@uneb.br

\begin{abstract}
Resumo
O presente trabalho analisa a utilização dos antimicrobianos em uma Unidade de Terapia Intensiva Neonatal, tendo em vista o seguimento farmacoterapêutico. Trata-se de um estudo de corte transversal realizado no período de junho a dezembro de 2018. Desse modo, observou-se que foram internados 124 pacientes na Unidade de Terapia Intensiva Neonatal. Deste total, 56 atendiam aos critérios de inclusão da pesquisa. Quanto às características da população estudada, a maioria dos pacientes foi do sexo masculino 60,7\%, a principal hipótese diagnóstica de infecção nos neonatos foi sepse precoce, correspondendo 55,3\%. Dos pacientes analisados 23 (41\%) tiveram hemocultura positiva, sendo que a principal bactéria isolada foi Staphylococcus coagulase negativa. A frequência dos antimicrobianos prescritos por paciente variou, sendo que: 5 (9\%) utilizaram apenas 1 antimicrobiano, 28 (50\%) utilizaram entre 2 e 3 antimicrobianos e 23 (41\%) utilizaram mais de 3 antimicrobianos. Os antimicrobianos mais prescritos foram gentamicina $33(15,7 \%)$, ampicilina $32(15,2 \%)$, amicacina $27(12,8 \%)$ e Oxacilina $23(11 \%)$. Em relação aos dias de tratamento, vancomicina e meropenem foram os medicamentos prescritos que tiveram maior duração, ambos 56 dias. Os dados apresentados demonstraram que o perfil da UTI neonatal estudada segue uma tendência mundial, fomentando a necessidade de desenvolver políticas de saúde que priorizem o uso racional de antimicrobianos.
\end{abstract}

Palavras-chave: Antimicrobianos; Neonatologia; Unidade de tratamento intensivo neonatal.

\begin{abstract}
This work analyzes the use of antimicrobials in a Neonatal Intensive Care Unit, based on the pharmacotherapeutic field. It's a cross-sectional study carried out from June to December 2018. It was observed that 124 patients were admitted to the Neonatal Intensive Care Unit. 56 of those attended the research inclusion criteria. As for the characteristics of the studied population, $60.7 \%$ were male, the main diagnostic hypothesis of infection in neonates was early sepsis, corresponding to $55.3 \% .23$ (41\%) of the studied patients had positive blood culture, and the main isolated bacterium was coagulase negative Staphylococcus. The frequency of antimicrobials prescribed per patient varied: $5(9 \%)$ used only 1 antimicrobial, 28 (50\%) used between 2 and 3 antimicrobials and $23(41 \%)$ used more than 3 antimicrobials. The most prescribed antimicrobials were gentamicin, $33(15.7 \%)$, ampicillin, $32(15.2 \%)$, amikacin, $27(12.8 \%)$ and Oxacillin, $23(11 \%)$. Regarding the days of treatment, vancomycin and meropenem were the prescription drugs that last the longest, both with 56 days. The data presented showed that the profile of the studied neonatal ICU follows a worldwide trend, encouraging the need to develop health policies that prioritize the rational use of antimicrobials.
\end{abstract}

Keywords: Antimicrobials; Neonatology; Neonatal intensive care unit. 


\begin{abstract}
Resumen
El presente trabajo analiza la utilización de los antimicrobianos en una Unidad de Terapia Intensiva Neonatal, con vistas al seguimiento farmacoterapéutico. Se trata de un estudio de corte transversal realizado en el período comprendido entre junio y diciembre de 2018. De este modo, se observó que 124 pacientes fueron internados en la Unidad de Terapia Intensiva Neonatal. De este total, 56 cumplían los criterios de inclusión de la investigación.. En cuanto a las características de la población estudiada, la mayoría de los pacientes fueron hombres 60,7\%, la principal hipótesis diagnóstica de infección en los neonatos fue sepsis precoz, correspondiendo 55,3\%. De los pacientes analizados 23 (41\%) tuvieron hemocultivo positivo, siendo que la principal bacteria aislada fue Staphylococcus coagulase negativa. La frecuencia de los antimicrobianos prescritos por paciente varió, siendo que: 5 (9\%) utilizaron sólo 1 antimicrobiano, $28(50 \%)$ utilizaron entre 2 y 3 antimicrobianos y $23(41 \%)$ utilizaron más de 3 antimicrobianos. Los antimicrobianos más recetados fueron gentamicina $33(15,7 \%)$, ampicilina $32(15,2 \%)$, amicacina $27(12,8 \%)$ y oxacilina $23(11 \%)$. En relación con los días de tratamiento, vancomicina y meropenem fueron los medicamentos recetados que tuvieron mayor duración, ambos 56 días. Los datos presentados demostraron que el perfil de la UCI neonatal estudiada sigue una tendencia mundial, fomentando la necesidad de desarrollar políticas de salud que prioricen el uso racional de antimicrobianos.
\end{abstract}

Palabras clave: Antimicrobianos; Neonatología; Unidad de tratamiento intensivo neonatal.

\title{
1. Introdução
}

Os antimicrobianos são medicamentos capazes de inibir ou eliminar o crescimento dos microrganismos devendo ser utilizados apenas para o tratamento de infecções microbianas que apresentam sensibilidade ao uso deste grupo farmacológico (Alves, et al., 2019). Uma das principais preocupações quanto ao seu uso é a resistência antimicrobiana, especialmente deflagrada por meio da utilização irracional dos medicamentos (Brasil, 2017). Segundo dados da Organização Mundial da Saúde, até o ano de 2050 ocorrerão cerca de 10 milhões de mortes a cada ano ocasionadas por doenças resistentes a medicamentos, o que poderá gerar danos comparáveis a crise financeira global de 2008 e 2009 (WHO, 2019).

A presença doenças infecciosas em recém nascidos ainda é muito comum mundialmente. Em países em desenvolvimento estima-se que a sepse neonatal ocorra em 15,4 casos para cada mil nascidos vivos, já nos Estados Unidos, a incidência varia de um a cinco para cada mil nascidos vivos (Goular et al., 2006). Nesse sentido, observa-se que cerca de 214.000 mortes por sepse neonatal no mundo são correlacionadas a cada ano a patógenos que se tornaram resistentes aos antimicrobianos prescritos (Laxminarayan et al., 2016).

O uso indiscriminado de antimicrobianos é frequente em todo o mundo, sendo um problema ainda mais sério nos países nos quais são de venda livre no comércio. Tal quadro se agrava com a inconsistência das medidas de controle no âmbito hospitalar (Rodrigues \& Bertoldi, 2010).

Os antimicrobianos são os medicamentos mais utilizados nas Unidades de Terapia Intensiva Neonatais (UTINs), compreendendo 3 dos 4 medicamentos habitualmente prescritos (Mukhopadhyay et al., 2019; Hsieh, et al., 2014). Em neonatologia, o uso indiscriminado de antimicrobianos pode ter uma grande repercussão devido o dinamismo farmacocinético dos neonatos e a complexidade terapêutica relacionada ao uso de antimicrobianos (Nunes, et al., 2017).

Neste sentido, neonatos possuem características próprias da infância que alteram a farmacocinética dos medicamentos, sendo, portanto, necessário maior vigilância e cuidado com a farmacoterapia nessa fase inicial, uma vez que muitos medicamentos são contraindicados para uso neonatal (Raposo, 2017).

Diante do exposto, a utilização racional dos antibióticos são pontos prioritários no contexto hospitalar, especialmente na neonatologia exigindo uma atenção especial quanto ao controle do uso dos antimicrobianos e padronização (Fernandes, 2006). O objetivo desse trabalho foi analisar a utilização dos antimicrobianos em uma Unidade de Terapia Intensiva Neonatal de um Hospital filantrópico de Salvador, tendo em vista o seguimento farmacoterapêutico.

\section{Metodologia}

Foi realizado um estudo transversal retrospectivo, de característica quantitativa, em uma UTI neonatal de um hospital 
filantrópico localizado em Salvador na Bahia no período de junho a dezembro de 2018. O hospital selecionado é classificado como um hospital de referência, atende especificamente crianças e adolescentes e possui mais de 30 especialidades.

Para elaboração do estudo utilizou-se como fonte de dados os prontuários médicos e formulários farmacêuticos. A avaliação dos isolados de bactérias foi realizada mediante dados registrados do laboratório. As variáveis adotadas foram relacionadas ao gênero do paciente, peso, motivo do internamento, antibiograma, cultura de bactérias e seguimento farmacoterapêutico. Também, utilizou-se a classificação Anatomical Therapeutic Chemical Classification (ATC), preconizada pela Organização Mundial de Saúde (OMS), para análise dos medicamentos prescritos quanto a suas propriedades farmacoterapêuticas. Para interpretação dos dados utilizou-se o método de análise quantitativa processados em programa estatístico SPSS ${ }^{\circledR}$.

Foram incluídos no estudo todos os pacientes internados na UTIN no período de junho a dezembro de 2018, que utilizaram antimicrobiano sistêmico durante o período do estudo e excluídos todos os pacientes internados que iniciaram o uso de antimicrobianos antes do início do período do estudo.

Para estruturação do estudo foi utilizado como base Pereira e colaboradores (2018). Já para embasar o trabalho utilizouse as bases de dados: bulário eletrônico da Agência Nacional de Vigilância Sanitária (ANVISA), MICROMEDEX 2.0 e Up to date, regulamentado pelo Food and Drug Administration (FDA), Scielo, PubMed, Medline, Guidelines.

O estudo foi aprovado pelo Comitê de Ética e Pesquisa da Maternidade Climério de Oliveira (MCO) - Universidade Federal da Bahia (UFBA) sob o parecer de aprovação número 3.604.99.

\section{Resultados}

Foram analisados 124 pacientes, deste total, 56 atendiam aos critérios de inclusão da pesquisa. Foram excluídos do estudo 68 pacientes, uma vez que iniciaram o uso de antimicrobianos antes do início do período do estudo.

A população estudada foi constituída, principalmente, de pacientes do sexo masculino 34 (60,7\%). A principal hipótese diagnóstica de infecção nos neonatos foi sepse precoce, correspondendo a 25 (44\%) pacientes. No Quadro 1 está representado as características sociodemográficas e clínicas dos pacientes.

Quadro 1. Características sociodemográficas e clínicas dos pacientes internados na UTIN do Hospital filantrópico de referência, município de Salvador, Bahia, 2020.

\begin{tabular}{|c|c|}
\hline VARIÁVEIS & N (\%) \\
\hline Sexo masculino & $34(60,7)$ \\
\hline Sexo feminino & $22(39,3)$ \\
\hline \multicolumn{2}{|c|}{ HIPÓTESE DIAGNÓSTICA } \\
\hline Sepse precoce & $25(44)$ \\
\hline Sepse tardia & $4(7,0)$ \\
\hline Outros & $28(49)$ \\
\hline
\end{tabular}

Fonte: Autores.

No quadro acima considerando as características sociodemográficas, houve uma predominância relevante do sexo masculino variável que se repete em outros estudos. Já em relação a hipótese diagnóstica a sepse precoce foi a mais prevalente, é necessário destacar que atualmente em UTIs neonatais esse diagnóstico é o mais presente. 
Das hemoculturas realizadas, 23 (41\%) pacientes tiveram hemocultura positiva, sendo que a principal bactéria isolada foi Staphylococcus coagulase negativa, equivalendo a $8(34,7 \%)$ pacientes. Os dados referentes as bactérias isoladas dos pacientes internados estão descritos no Quadro 2.

Quadro 2. Bactérias isoladas dos pacientes internados na UTIN do Hospital filantrópico de referência, município de Salvador, Bahia, 2020.

\begin{tabular}{|c|c|}
\hline BACTÉRIA ISOLADA & $\mathbf{N}(\mathbf{\%})$ \\
\hline Hemoculturas positivas & $23(41)$ \\
\hline Staphylococcus coagulase negativa & $8(34,7)$ \\
\hline Klebsiella pneumoniae & $4(17,3)$ \\
\hline Staphylococcus aureus & $3(13)$ \\
\hline Acinetobacter baunannii & $3(13)$ \\
\hline Outros & $5(22)$ \\
\hline
\end{tabular}

Fonte: Autores.

De acordo com o Quadro 2, dos 56 pacientes 23 tiveram hemocultura positiva, sendo que todos pacientes realizaram hemocultura. Alguns fatores podem mascarar a hemocultura, como a exemplo do uso de antimicrobianos prévio, coletas realizadas incorretamente, armazenamento errôneo do material, entre outros, nessa pesquisa esses fatores não foram analisados. Entre as bactérias isoladas a Staphylococcus coagulase negativa prevaleceu, essa bactéria tem se mostrado muito comum em Unidades de Terapia Intensiva e está correlacionada com a sepse neonatal.

A frequência dos antimicrobianos prescritos por paciente variou, sendo que: 5 (9\%) pacientes utilizaram apenas 1 antimicrobiano, 28 (50\%) utilizaram entre 2 e 3 antimicrobianos e 23 (41\%) utilizaram mais de 3 antimicrobianos (Quadro 3).

Quadro 3. Frequência de antimicrobianos prescritos para 56 paciente internados na UTIN do Hospital filantrópico de referência, município de Salvador, Bahia, 2020.

\begin{tabular}{|c|c|c|}
\hline ANTIMICROBIANOS POR PACIENTE & $\mathbf{N}^{\mathbf{0}}$ & \multicolumn{1}{|c|}{} \\
\hline 1 antimicrobiano & 5 & 50 \\
\hline 2 e 3 antimicrobianos & 28 & 51 \\
\hline Mais de 3 antimicrobianos & 23 & 41 \\
\hline
\end{tabular}

Fonte: Autores.

Como representado no quadro 3, dos 56 pacientes 28 deles, ou seja, metade do total consumiram de 2 a 3 antimicrobianos durante o internamento, pode-se perceber que 23 pacientes que representa $41 \%$ do total consumiu mais de 3 antimicrobianos, valor que também é relevante e pode estar associado ao alto consumo.

Em relação ao perfil dos antimicrobianos prescritos (Quadro 4), 210 antimicrobianos foram administrados, ficando em média 3,75 por paciente, sendo que os que tiveram maior destaque foram: gentamicina 33 (15,7\%), ampicilina 32 (15,2\%), 
amicacina $27(12,8 \%) 3$ oxacilina $23(11 \%)$. Quanto aos dias de tratamento, os mais duradouros foram realizados com vancomicina e meropenem (ambos 56 dias).

Quadro 4. Perfil dos antimicrobianos prescritos para 56 pacientes internados na UTIN do Hospital filantrópico de referência, município de Salvador, Bahia, 2020.

\begin{tabular}{|c|c|c|}
\hline ANTIMICROBIANO & FREQUÊNCIA & DIAS DE TRATAMENTO \\
\hline Gentamicina & 33 & 2 a 12 \\
\hline Ampicilina & 32 & 2 a 10 \\
\hline Amicacina & 27 & 1 a 10 \\
\hline Oxacilina & 23 & 1 a 14 \\
\hline Vancomicina & 22 & 2 a 56 \\
\hline Cefepime & 18 & 2 a 14 \\
\hline Meropenem & 17 & 2 a 56 \\
\hline Metronidazol & 8 & 2 a 22 \\
\hline Polimixina B & 8 & 2 a 22 \\
\hline Cefuroxima & 5 & 1 a 2 \\
\hline Sulfa+trimetopim & 4 & 10 a 14 \\
\hline Cefazolina & 3 & 1 a 2 \\
\hline Piperacilina4+tazobactam & 3 & 2 a 12 \\
\hline Ciprofloxacino & 2 & 2 a 9 \\
\hline Clindamicina & 2 & 1 \\
\hline Teicoplanina & 2 & 3 \\
\hline Cefalexina & 1 & 10 \\
\hline
\end{tabular}

Fonte: Autores.

O Quadro 4 demostra que os diferentes tipos de antimicrobianos foram prescritos 210 vezes, sendo que desses a gentamicina foi a mais prescrita, e considerando os dias de tratamento os antimicrobianos vancomicina e meropenem foram os mais utilizados variando de 2 a 56 dias de tratamento.

Foram prescritos 17 tipos diferentes de antimicrobianos divididos nos 5 grupos mais frequentes de acordo com a classificação ATC como representado na Figura 1. 
Figura 1. Principais grupos de antimicrobianos prescritos de acordo com a classificação Anathomical Therapeutic Chemical.

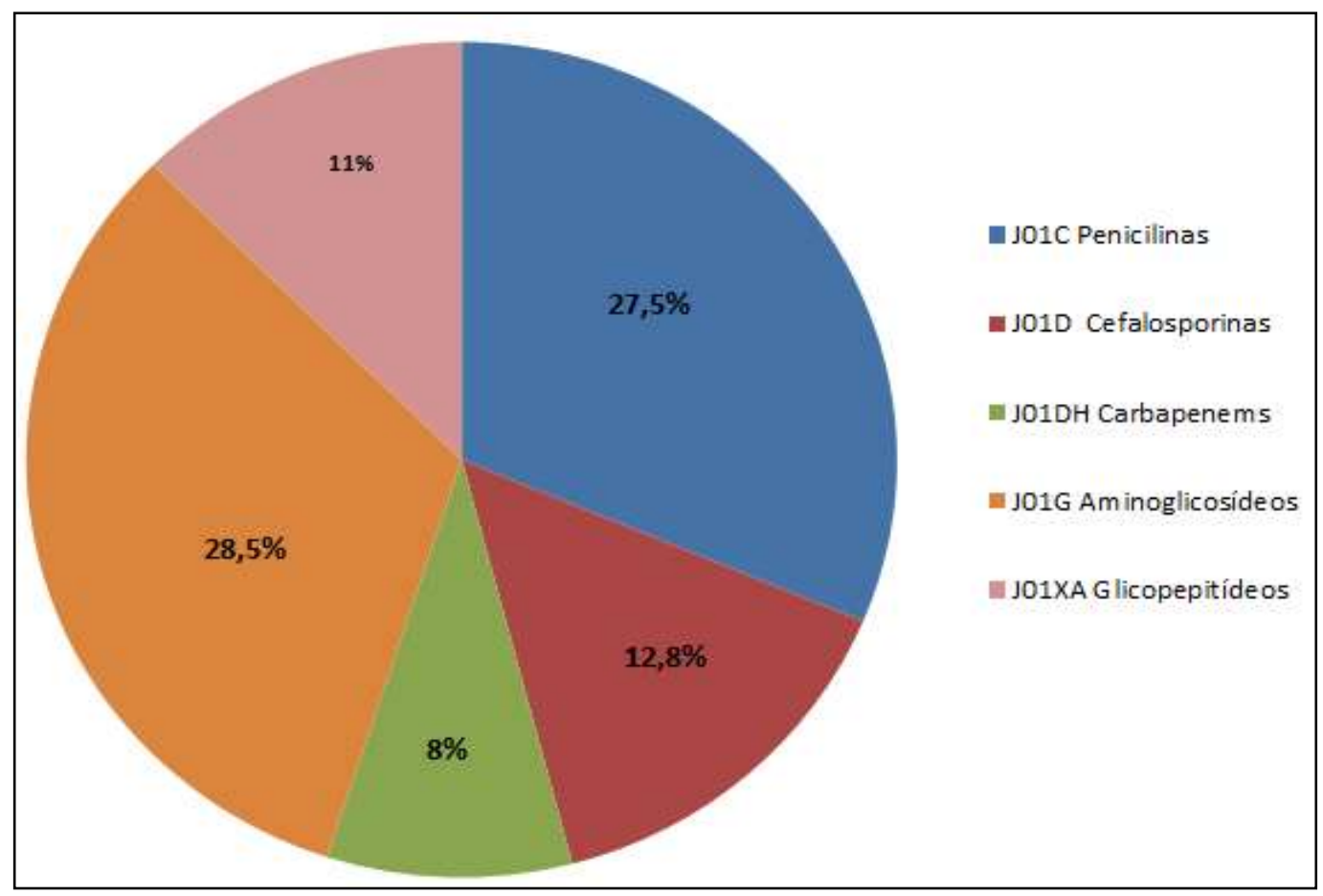

Fonte: Autores.

De acordo com a Figura 1 a classe de antimicrobianos mais prescrita de acordo com a classificação ATC foi a dos aminoglicosídeos, representando $28,5 \%$ do total.

\section{Discussão}

Considerando as características da população desse estudo, constatou-se maior frequência do gênero masculino, achado semelhante a estudos realizados em Unidades de Terapia Intensiva Neonatal em Santa Catarina (Rodrigues \& Belham, 2017) e em São Paulo (Oliveira et al., 2015).

Verificou-se que a principal hipótese diagnóstica de infecção foi a sepse precoce. Estudo desenvolvido por Castro (2017) demonstrou que tantos países em desenvolvimento, quanto países desenvolvidos, possuem estimativas de sepse alarmantes. A nível global, a ocorrência de sepse neonatal "varia de 7,1 a 38 por 1000 nascidos vivos na Ásia, de 6,5 a 23 por 1000 nascidos vivos na África e de 3,5 a 8,9 por 1000 nascidos vivos na América do Sul e Caribe (Castro, 2017).

Entre os microrganismos isolados, encontrou-se o Staphylococcus coagulase negativa como o mais comum. Assim, trata-se de uma bactéria gram-positiva, que se destaca em sepse neonatal. Estudos demonstram a elevada incidência destes microrganismos na população neonatal. Um estudo conduzido no nordeste brasileiro demostrou que 63,4\% dos microrganismos isolados em uma UTIN, de junho de 2015 a junho de 2017, eram Staphylococcus coagulase negativa (Souza et al., 2019). Dados internacionais também relatam a mesma realidade, no Irã, um estudo elaborado por Mahalley, et al. (2018) demostrou que o Staphylococcus coagulase negativa prevaleceu entre os microrganismos isolados $37,31 \%$. Também, outro estudo realizado na Polônia o Staphylococcus coagulase negativa com o mais incidente (36,1\%) (Sadowska-Krawczenko et al.,2012).

No presente estudo, a maioria dos pacientes internados que utilizaram antimicrobianos recebeu de dois a três antimicrobianos, seguido, mais de três antimicrobianos, enquanto que uma pequena parcela recebeu apenas um. Esses dados se assemelham com o estudo realizado em um Hospital público do Pará, por Silva (2012). Neste sentido, práticas que visam o 
sinergismo da ação farmacológica, utilizam a associação de fármacos com o objetivo de obter melhora terapêutica dos pacientes (Locateli, 2017).

A ampicilina, amicacina e gentamicina estão entre os medicamentos que foram mais prescritos nesse estudo. De acordo com os protocolos da Sociedade Brasileira de Pediatria esses antimicrobianos são os mais utilizados em neonatologia, tal quadro justifica-se para fins de tratamento empírico, em UTIN, em razão da baixa indução resistência, bem como, no que se refere à amicacina, a considerável sensibilidade dos bastonetes gram-negativos, e também em função de seus custos reduzidos e disponibilidade considerável (Júca, 2016)

Partindo-se dos riscos inerentes ao uso de aminoglicosídeos, referentes à ototoxicidade e nefrotóxicas, e o consequente tipo de cuidado adotado em função dessa situação, pode-se fazer um paralelo no tocante a outras drogas potencialmente ototóxicas e nefrotóxicas, que também exigem as mesmas precauções (Calil \& Caldas, 2012).

Em relação ao tempo de tratamento, a vancomicina e o meropenem se destacaram, o tempo de utilização foi de 2 a 56 dias. O uso prolongado de antimicrobianos de amplo espectro, especialmente em lactentes de alto risco, tem sido apontado como fator de risco para a ocorrência de enterocolite necrosante, displasia broncopulmonar e candidíase invasiva, o que pode potencializar o agravamento do quadro clínico, com evolução para o óbito (Sánchez et al.,2016; Cantey et al.,2016).Nesse sentido, o uso de antimicrobianos de amplo espectro, em especial vancomicina e carbapenêmicos, deve ser avaliado criteriosamente, devido ao difícil manuseio em casos de resistência a esses medicamentos.

A preocupação com a resistência bacteriana vem sendo relatada em vários estudos e pesquisas significativas direcionadas a descobertas de novos antimicrobianos de amplo espectro ainda são escassas. Desse modo, na tentativa de reduzir a resistência bacteriana, diferentes países estão implementando políticas de uso sustentável de antimicrobianos (Locateli, 2017).

Assim a análise da utilização de antimicrobianos em neonatologia é de suma importância, pois a utilização irracional de antimicrobianos representa um problema de saúde pública. Logo, faz-se necessário desenvolver ações de saúde que priorizem o seu uso de forma racional, a exemplo da implantação do programa de gerenciamento de antimicrobianos "Antimicrobial Stewardship Program (ASP)" que consiste em um conjunto de intervenções coordenadas, com a função de melhorar e medir o uso adequado dos antimicrobianos por meio da promoção da seleção otimizada do regime antimicrobiano ideal (Barlam et al., 2016).

\section{Conclusão}

Os dados apresentados nesse estudo demonstram que o perfil da UTIN estudada segue uma tendência mundial, o que acaba gerando uma inquietude frente à realidade apresentada. Afinal, demonstrou-se o crescimento de microrganismos antes incomuns em neonatos internados em UTIN, além do alto consumo e longo tratamento com antimicrobianos.

Mais da metade dos pacientes internados que atendiam os critérios do estudo tiveram hemocultura negativa, sugerindo possíveis falhas durante o processo de coleta do material sanguíneo ou na indicação terapêutica, uma vez que os pacientes seguiam utilizando os antimicrobianos empiricamente.

A criação de protocolos de diagnóstico e tratamento para determinados quadros clínicos, bem como a adoção de práticas que guiem a utilização empírica dos antimicrobianos, são formas sustentáveis de redução da pressão seletiva de microrganismos resistentes ao uso de antimicrobianos.

Considerando que os antimicrobianos são medicamentos essenciais no contexto hospitalar, além de estarem entre os mais utilizados, faz-se necessário desenvolver políticas de saúde que priorizem o seu uso de forma racional. Além disso, a Comissão de Controle de Infecções Hospitalar deve atuar regularmente, proporcionando educação continuada aos profissionais de saúde. 
Em suma, os resultados do estudo retratam a realidade de uma UTIN e consequentemente instigam a realização de novos estudos, uma vez que possibilita traçar perfis de consumo de antimicrobianos em diferentes Unidades de Terapia Intensiva Neonatais e fazer comparações dos perfis etiológicos dos microrganismos. Também, pode influenciar em mudança de ações pelos profissionais de saúde além de poder impulsionar os gestores a realização de novas intervenções direcionadas à diminuição da resistência antimicrobiana.

\section{Referências}

Alves, L. S., da Silva, P. L. N., Fonseca, J. R., Vaz, M. D. T., \& Santo, L. R. E. (2019). Análise de interação medicamentosa de prescrições médicas contendo antimicrobianos de uma drogaria privada de Minas Gerais. JMPHC| Journal of Management \& Primary Health Care| ISSN $2179-6750,10$.

Barlam, T. F., Cosgrove, S. E., Abbo, L. M., MacDougall, C., Schuetz, A. N., Septimus, E. J., \& Trivedi, K. K. (2016). Implementing an antibiotic stewardship program: guidelines by the Infectious Diseases Society of America and the Society for Healthcare Epidemiology of America. Clinical infectious diseases, 62(10), e51-e77.

Brasil. Ministério da Saúde. (2017) Agência Nacional de Vigilância Sanitária. Diretriz Nacional para Elaboração de Programa de Gerenciamento do Uso de Antimicrobianos em Serviços de Saúde. Brasília: Ministério da Saúde.

Calil, R., \& Caldas, J. M. S. (2012). Uso racional e seguro de antibióticos em Neonatologia. Sociedade Brasileira de Pediatria.

Cantey, J. B., Wozniak, P. S., Pruszynski, J. E., \& Sánchez, P. J. (2016). Reducing unnecessary antibiotic use in the neonatal intensive care unit (SCOUT): a prospective interrupted time-series study. The Lancet Infectious diseases, 16(10), 1178-1184.

Castro, R. S. A. P. D. (2017). Análise da sepse neonatal tardia em prematuros de muito baixo peso após a implantação do protocolo de sepse na unidade. Fernandes, F. M. N. (2006). Prevalência da Infecção Hospitalar em Unidades de Neonatologia de Salvador e Região Metropolitana.

Goulart, A. P., Valle, C. F., Dal-Pizzol, F., \& Cancelier, A. C. L. (2006). Risk factors for early-onset neonatal sepsis in Brazilian public hospital short-title: earlyonset neonatal sepsis. Revista Brasileira de terapia intensiva, 18(2), 148-153.

Hsieh, E. M, Hornik, C. P, Clark, R. H, Laughon, MM, Benjamin Jr, D. K, \& Smith, P. B (2014). Uso de medicamentos na unidade de terapia intensiva neonatal. American Journal of Perinatology, $31(9), 811$.

Jucá, F. L. (2016). O uso de antimicrobianos em Unidade de Terapia Intensiva Pediátrica (Doctoral dissertation, Universidade de São Paulo).

Laxminarayan, R., Matsoso, P., Pant, S., Brower, C., Røttingen, J. A, Klugman, K., \& Davies, S. (2016). Acesso a antimicrobianos eficazes: um desafio mundial. The Lancet, 387 (10014), 168-175.

Locatelli, D. L. (2017). Perfil de antimicrobianos utilizados em uma unidade de tratamento intensivo neonatal de um hospital materno infantil.

Mahallei, M., Rezaee, M. A., Mehramuz, B., Beheshtirooy, S., \& Abdinia, B. (2018). Clinical symptoms, laboratory, and microbial patterns of suspected neonatal sepsis cases in a children's referral hospital in northwestern Iran. Medicine, 97(25).

Mukhopadhyay, S., Wade, K. C., \& Puopolo, K. M. (2019). Drugs for the Prevention and Treatment of Sepsis in the Newborn. Clinics in perinatology, 46(2), 327-347.

Nunes, B. M., Xavier, T. C., \& Martins, R. R. (2017). Problemas relacionados a medicamentos antimicrobianos em unidade de terapia intensiva neonatal. Revista Brasileira de Terapia Intensiva, 29(3), 331-336.

Oliveira, C. D. S., Casagrande, G. A., Grecco, L. C., \& Golin, M. O. (2015). Perfil de recém-nascidos pré-termo internados na unidade de terapia intensiva de hospital de alta complexidade. ABCS Health Sci.

Pereira, A. S., Shitsuka, D. M., Parreira, F. J., \& Shitsuka, R. (2018). Metodologia da pesquisa científica.

Raposo, M. J. M. M. (2017). Abordagem farmacológica em Neonatologia: órfãos terapêuticos (Master's thesis).

Rodrigues, F. D. A., \& Bertoldi, A. D. (2010). Perfil da utilização de antimicrobianos em um hospital privado. Ciência \& Saúde Coletiva, $15,1239-1247$.

Rodrigues, V. B. M., \& Belham, A. (2017). Perfil dos recém-nascidos admitidos na UTI neonatal do hospital Santo Antônio, Blumenau/SC, entre 20142016. Arquivos Catarinenses de Medicina, 46(4), 43-49.

Sadowska-Krawczenko, I., Jankowska, A., \& Kurylak, A. (2012). Healthcare-associated infections in a neonatal intensive care unit. Archives of Medical Science: AMS, 8(5), 854

Sánchez, P. J., Moallem, M., Cantey, J. B., Milton, A., \& Michelow, I. C. (2016). Empiric therapy with vancomycin in the neonatal intensive care unit: let's" get smart" globally!. Jornal de Pediatria, 92(5), 432-435.

Silva, E. R. M. (2012). Análise do perfil das prescrições de antimicrobianos na clínica médica de um hospital público do Pará. Revista Brasileira de Farmácia Hospitalar e Serviços de Saúde, 3(2). 
Research, Society and Development, v. 10, n. 1, e29810111794, 2021

(CC BY 4.0) | ISSN 2525-3409 | DOI: http://dx.doi.org/10.33448/rsd-v10i1.11794

Sousa, N. A., Coelho, C. G. V., Mesquita, C. H. S., Pires, F. G. B., Rosa, P. B., \& Brito, I. L. P. (2019). Sepse neonatal-perfil microbiológico e sensibilidade antimicrobiana em um hospital no Nordeste do Brasil. Revista Bras. de análises clínicas, 51(1), 46-51.

World Health Organization (2019). Antimicrobial stewardship programmes in health-care facilities in low-and middle-income countries: a WHO practical toolkit. 